\title{
Seguimiento de Egresados del Programa de Maestría en Enfermería. 2o cohorte generacional
}

\author{
2nd Generational cohort nursing program \\ graduates follow-up
}

Mtra. Martha Lilia Bernal Becerril*, Mtra. Gandhy Ponce Gómez** y Mtra. Laura Morán Peña***

*Maestra en Enfermería con orientación en Educación en Enfermería, Profesor de Carrera Asociado B. de T.C. Unidad de Investigación. ENEO-UNAM, **Maestra en Enfermería con orientación en Educación en Enfermería, Profesor de Carrera Asociado B. de T.C. Unidad de Investigación. ENEO-UNAM y *** Maestra en Enfermería en Educación Superior, Profesor de Carrera Titular B. Secretaria de Planeación. ENEO-UNAM.

\section{Resumen}

Estudios de Seguimiento de Egresados (ESE) tienen como fines la evaluación curricular, reconocer la pertinencia de la formación recibida y la inserción laboral; también, proporcionan indicadores sobre la eficiencia de las instituciones educativas. Una evaluación que permita reconocer la perspectiva de los alumnos, enriquece a futuras generaciones, en la medida que posibilite ubicar y reconocer las debilidades y fortalezas. Metodología: Estudio transversal correlacional en egresados de la $2^{a}$ generación del Programa de Maestría en Enfermería $(\mathrm{N}=12)$ empleando un instrumento autoadministrado, con escala Likert. En el diseño del instrumento se retomaron aportaciones de Viniegra, (1997); Fresán, (2000); Loredo y Grijalva, (2000) y Morán, (2002). Resultados: La satisfacción general percibida por el Programa de Maestría en Enfermería (PME) alcanza una media de 4.5; lo que indica alta calidad del programa y alta satisfacción por los estudios cursados. La correlación entre variables alcanzó una fuerza alta entre las variables contenidas en las dimensiones entrada - proceso - resultado. Discusión: Actualmente se realizan esfuerzos por generar una visión global de la evaluación educativa, que implique la consideración del ambiente interno y externo, los subsistemas y escenarios de trabajo, procesos, actores, insumos y producto, así como la identificación y análisis del impacto que producen (Amestoy, 2002). Conclusiones: La formación de recursos humanos con calidad requiere de condiciones para el aprendizaje que permitan el dominio de los conocimientos, condiciones de infraestructura y profesores calificados para que los egresados sean capaces de responder a los retos que plantea el ambiente.

Palabras clave: Seguimiento de Egresados, Maestría, Calidad. 


\section{Abstract}

The follow-up studies of graduates (ESE) have the goal of curricula evaluation, recognizing the academic upbringing pertinence received and the later work insertion to the post-grade studies: they also give a series of indicators about the efficiency of the educational institutions. Having a model or instrument that allow to carry out an evaluation of Programs at post-grade level form the students perspective will enrich largely future generations, and as far as can be, make possible locate and recognize weaknesses and strengths to make them solid enough to constitute a reference from this investigation. Metodology: It is a transversal correlational study on the generation 2005-2006 graduates population of the UNAM Nursing Mastery Program $(N=12)$ through a self-applied instrument, measured with a Likert-like scale. For the instrument's design, the contributions of Viniegra (1997), Fresan (2000), Loredo and Grijalva (2000) and Moran (2002) were retaken. Results: The perceived general satisfaction about professional upbringing received by PME reaches a media of 4.5 with points to a high quality perception of the program and a high satisfaction with the studies finished. Regarding co-relation between variables it points that there is a high co-relation strength between variables contained in the dimensions of entry-process- result. Discussion. Nowadays, efforts for generating a global educational evaluation vision are being made, implying incorporating the most possible variable number, considering an internal and external environment, the different subsystems and working sceneries, the different processes, the actors, assets and product, as well as identification and analysis of different types of impacts they produce (Amestoy, 2002). Conclusions. Quality Human resources creation requires conditions for learning that allow the mastery of knowledge, infrastructure conditions and qualified professors in order the graduates to be capable to answer the challenges the environment places, and is one of the tasks to which the efforts of high education efforts must be targeted.

Key Words: Graduates follow-up. Mastery. Quality

\section{INTRODUCCIÓN}

Los estudios de seguimiento de egresados (ESE) son una estrategia curricular para evaluar el desempeño y desarrollo profesional que a su vez, orientan la toma de decisiones en el plan curricular; además tienen como fines la evaluación curricular, reconocer la pertinencia de la formación académica recibida y la inserción laboral posterior a los estudios de posgrado; también, proporcionan una serie de indicadores sobre la eficiencia de las instituciones educativas".

La evaluación sistémica-integral, es una combinación de los planteamientos de Stufflebeam (1989) y Stake (1987).
En dicha propuesta Stufflebeam incorpora componentes como entrada, procesos y productos. Por su parte Stake, con su perspectiva de "la evaluación respondiente", pone su acento en la participación de los involucrados y sus opiniones ${ }^{2}$. Es a partir de esta visión que se pretende además de dar seguimiento a la situación de los egresados, llevar a cabo una evaluación integral-sistémica en que se estudia la percepción del maestrando acerca de la calidad del programa desde el paradigma sistémico (entrada - proceso - resultado). (Ver figura 1)

\section{VISIÓN INTEGRAL SISTÉMICA DE LA EVALUACIÓN EDUCATIVA}

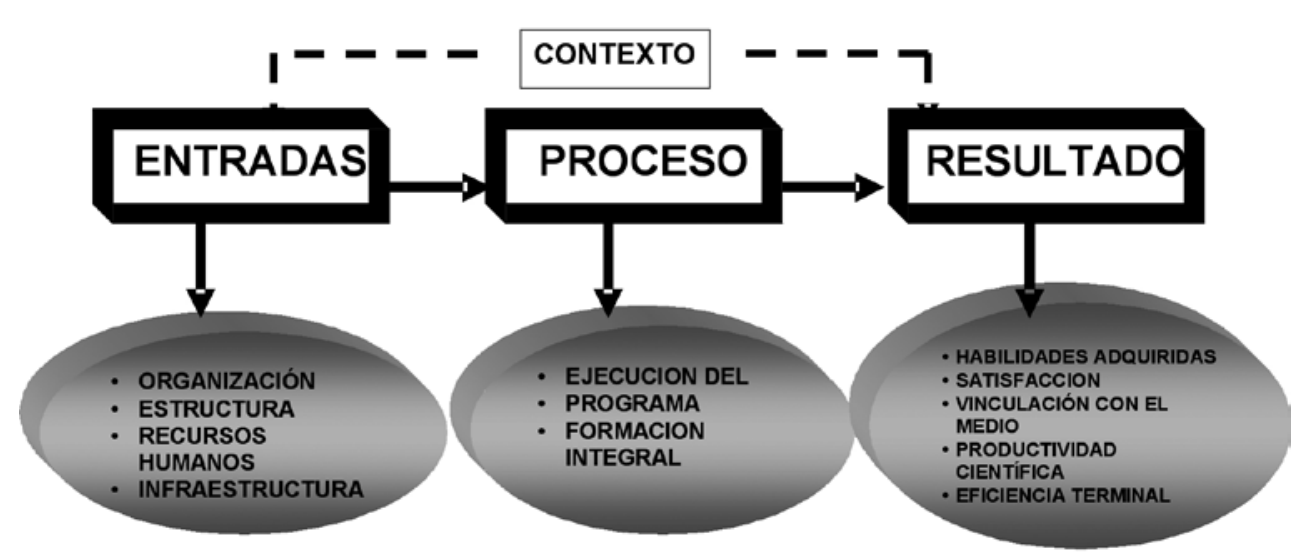

FIGURA NO. 1 
La práctica educativa, de acuerdo con Fierro, Fortoul y Rosas, debe ser entendida como una praxis social, objetiva e intencional en la que intervienen los significados, las percepciones y las acciones de los agentes implicados en el proceso - docentes, alumnos y autoridades educativas, así como los aspectos político - institucionales, administrativos y normativos y en la que la identificación de limitaciones y deficiencias en el aprendizaje debe orientarse hacia el planteamiento de alternativas que les permitan realizar una enseñanza y un aprendizaje de excelencia ${ }^{3}$.

Es así como este estudio pretende: a) Valorar la calidad de la oferta de la formación profesional de los egresados, en función de su movilidad en el mercado laboral, la productividad académica y la satisfacción por la formación recibida. b) Analizar la percepción que tiene el maestrando de la $2^{a}$ generación acerca de la formación profesional ofertada por el PME en función de una evaluación integral sistémica que describa las características de entrada, proceso y resultado. c) Identificar si hay asociación entre las variables entrada, proceso y resultado y d) Describir el nivel de satisfacción general que manifiesta el egresado del PME de la generación 2005 - 2006.

\section{El seguimiento de egresados como estrategia de evaluación}

Se denominan seguimiento de egresados a aquellas propuestas metodológicas que tienen por objetivo conocer el destino laboral, ocupacional o escolar de quienes han salido del mismo "ciclo, nivel, subsistema, modalidad, institución o programa educativo"4; y se encuentran circunscritos al área de las relaciones del sector educativo y social, en especial en la línea económica que por su complejidad esta fundamentada en diversas corrientes teóricas para poder llevar a cabo el análisis de las actividades profesionales de los graduados, los resultados de su formación, su campo de acción, su nivel de ingresos, su ubicación y formación académica posteriores al egreso y las exigencias y demandas a las que están sometidos en el trabajo¹.

Entre los beneficios que reportan los ESE se encuentran:

- Constituyen una alternativa para la autoevaluación y la planeación de procesos de mejora de las instituciones educativas.

- Son una herramienta para analizar los caminos que siguen los profesionales.

- Proporcionan elementos de diagnóstico de la realidad, ayuvando a la reflexión sobre los procesos, fines y valo- res de las instituciones de educación superior y de posgrado.

- Retroalimentar los programas de formación de profesionales e investigadores en las instituciones de educación.

- Constituyen uno de los indicadores más confiables de la pertinencia, suficiencia y actualidad de los programas educativos que sustentaron su formación.

- Contribuyen indirectamente a evidenciar la calidad de la planta académica de las instituciones educativas, la pertinencia y actualidad de sus planes y programas de estudio y la idoneidad de sus estrategias pedagógicas.

- Permite conocer las nuevas exigencias que plantea el ejercicio profesional a los egresados como consecuencia de las transformaciones económicas, sociales y tecnológicas, para su incorporación oportuna en los planes y programas de estudios y el diseño de programas de educación continua pertinentes para la actualización de profesionales en ejercicio7.

\section{METODOLOGÍA}

Se trata de un estudio transversal correlacional efectuado en el total de la población de egresados de la generación 2005 - 2006 ( $\mathrm{N}=12)$ a través de un instrumento autoadministrado y anónimo, medido con una escala tipo Likert, donde 1 era totalmente en desacuerdo y 5 totalmente de acuerdo, constituyéndose en una escala de percepción de la calidad donde de 4.1 a 5 se tiene alta calidad o satisfacción, 3.1. A 4 adecuada calidad o satisfacción y de 2.1 a 3 regular calidad o satisfacción. Para el diseño del instrumento se retomaron las aportaciones de Viniegra ${ }^{5}$, Fresán ${ }^{6}$, Loredo y Grijalva ${ }^{7}$, y Morán ${ }^{8}$.

Las evaluaciones se llevaron a cabo al final de cada semestre, antes de que los alumnos recibieran calificaciones con el fin de evitar tendencias.

\section{RESULTADOS.}

El 100\% de los egresados de la generación 2005- 2006 corresponde a mujeres; el $42 \%$ casadas y el $33 \%$ de solteras. Con respecto a estudios previos se encuentra que el 25\% cuenta con algún posgrado (especialidad), mientras que el $75 \%$ tiene nivel de licenciatura. Tres cuartas partes de la población labora en un solo empleo y el 17\% en dos, mientras que respecto a las actividades que desempeña se tiene que el $75 \%$ labora en una institución de salud con funciones de tipo asistencial y $25 \%$ en instituciones educativas. La ubicación de su lugar de trabajo es de 83\% en el D.F. y el 17\% en otro estado de la República. 
La movilidad laboral muestra que ha habido cambio en el $25 \%$ de la población después de cursado el Programa de Maestría. Se detectó que la predominancia de las actividades en el primer empleo son de tipo asistencial (41\%), administrativas (25\%) y las de docencia e investigación (17 y $8.3 \%$ respectivamente).

Respecto al tiempo que transcurre entre el egreso de la licenciatura y el ingreso al posgrado, el $42 \%$ sólo espero un lapso menor a 4 años y el 33\% tardó entre 5 y 8 años, el resto más de 13 años para cursar la maestría.

Refiriéndose a los estudios de interés a futuro, refieren que cursarán el doctorado (33\%) y la especialidad (33\%).

El avance de la producción de su tesis muestra que el $60 \%$ de los casos tiene el $60 \%$ o menos de avance en su trabajo final y el $40 \%$ han logrado un avance del $70 \%$ más. El 50\% de la población considera graduarse en menos de un año y el resto en 1 año. Es importante hacer notar que el $17 \%$ de la población tiene dificultades para titularse debido a las actividades en su trabajo.

Refiriéndose a la producción científica, ésta muestra la presentación de trabajos a nivel nacional o internacional donde el $25 \%$ de los egresados ha participado en cursos como ponentes y el $17 \%$ como asistente, el 50\% ha asistido a congresos, y ha publicado en revistas o memorias a nivel internacional.

Desde el punto de vista sistémico los resultados muestran que la calidad en la dimensión Entrada (donde se consideró la organización, estructura, recursos humanos e infraestructura con la que se cuenta para la implementación y desarrollo del PME), se observó una media de 4.6; para la dimensión Proceso, (donde se tomó en cuenta: la ejecución del programa y la formación integral) se obtuvo una media de 4.8; y para la dimensión Resultado, (considerando las habilidades adquiridas, satisfacción, vinculación con el medio, productividad científica y la eficiencia terminal) se obtuvo una media de 4.5.

La satisfacción general percibida acerca de la formación profesional recibida por el PME alcanza una media de 4.5; lo que nos habla de una percepción de alta calidad del programa y alta satisfacción por los estudios cursados. (Ver cuadro 2)

En cuanto a la correlación entre variables se obtuvo que existe una fuerza de correlación alta entre las variables contenidas en las dimensiones entrada - proceso resultado (entrada - proceso $\mathrm{rP}=.881$, entrada - resultado $\mathrm{rP}=.833$ y proceso - resultado $\mathrm{r}=.863$; en todas con probabilidad de .000).
CUADRO 2.

Índice de Satisfacción por dimensión

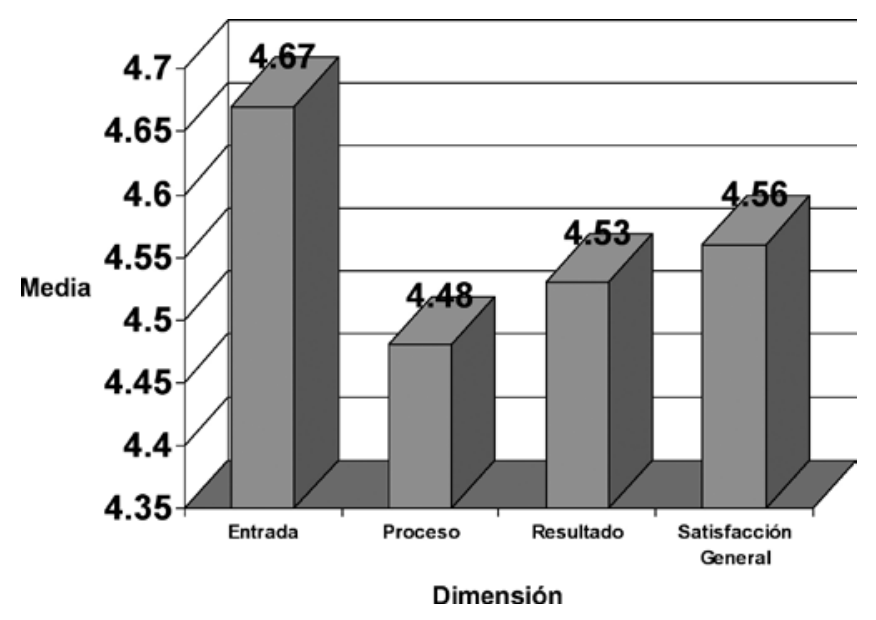

Respecto a la confiabilidad del instrumento, se obtuvo un alfa de Cronbach de .9758 lo que puede dar cuenta de la consistencia interna de los ítems.

\section{DISCUSIÓN}

La presente investigación coincide en el alto nivel de satisfacción con el referido en el estudio de Ramírez y Tovar ${ }^{9}$ en Egresados de las especializaciones en enfermería de la Universidad del Valle, así como el alto nivel de satisfacción con la formación recibida y la satisfacción con la formación personal. Acerca del incremento de la productividad científica de los egresados al compartir sus hallazgos de investigación en artículos de revistas o textos, se asemejan los hallazgos con lo reportado por Martínez y Salazar ${ }^{10}$, quienes aseguran que sus entrevistadas se encontraban participando en proyectos de investigación, algunas inclusive eran responsables de dichos proyectos. El estudio del nivel de calidad percibido, así como la visión desde el paradigma sistémico permite a los programas de posgrado plantear planes y acciones conducentes a integrar los comentarios realizados por los egresados y a diseñar acciones dirigidas a la búsqueda de la excelencia.

\section{CONCLUSIÓN}

La formación de recursos humanos con calidad requiere de condiciones para el aprendizaje que permitan el dominio de los conocimientos, condiciones de infraestructura y profesores calificados para que los egresados sean capaces de responder a los retos que plantea el ambiente, y es una de las tareas a la que deben dirigir sus esfuerzos las instituciones de educación superior. 
Es trascendente contar con un elemento más para evaluar el Programa de Maestría en Enfermería, a través de un modelo sistémico integral y contar con un instrumento que responda a tal visión, dentro del contexto del posgrado, y sobre todo tomando en cuenta la perspectiva del propio alumno, lo que fortalecerá tanto al Programa como a las futuras generaciones.

Se cuenta con una evaluación desde la perspectiva de los alumnos de la segunda generación que permitirá la retroalimentación y fortalecimiento del Programa de Maestría en Enfermería de la UNAM, considerando que la percepción de la calidad y la satisfacción por los estudios cursados son altas en ambos casos.

El seguimiento de egresados es una estrategia curricular para evaluar el desempeño y desarrollo profesional de quienes se gradúan en una carrera, así como para identificar la adecuación que hay entre la formación recibida y los perfiles profesional y ocupacional definidos con el aporte de datos significativos que, a su vez, sirven para tomar decisiones en los programas educacionales, además deben considerar las exigencias científico-técnicas, humanísticas y sociales cambiantes y las necesidades del mundo del trabajo y que los egresados, las investigaciones y la producción intelectual se consideran los logros mas importantes de las universidades.

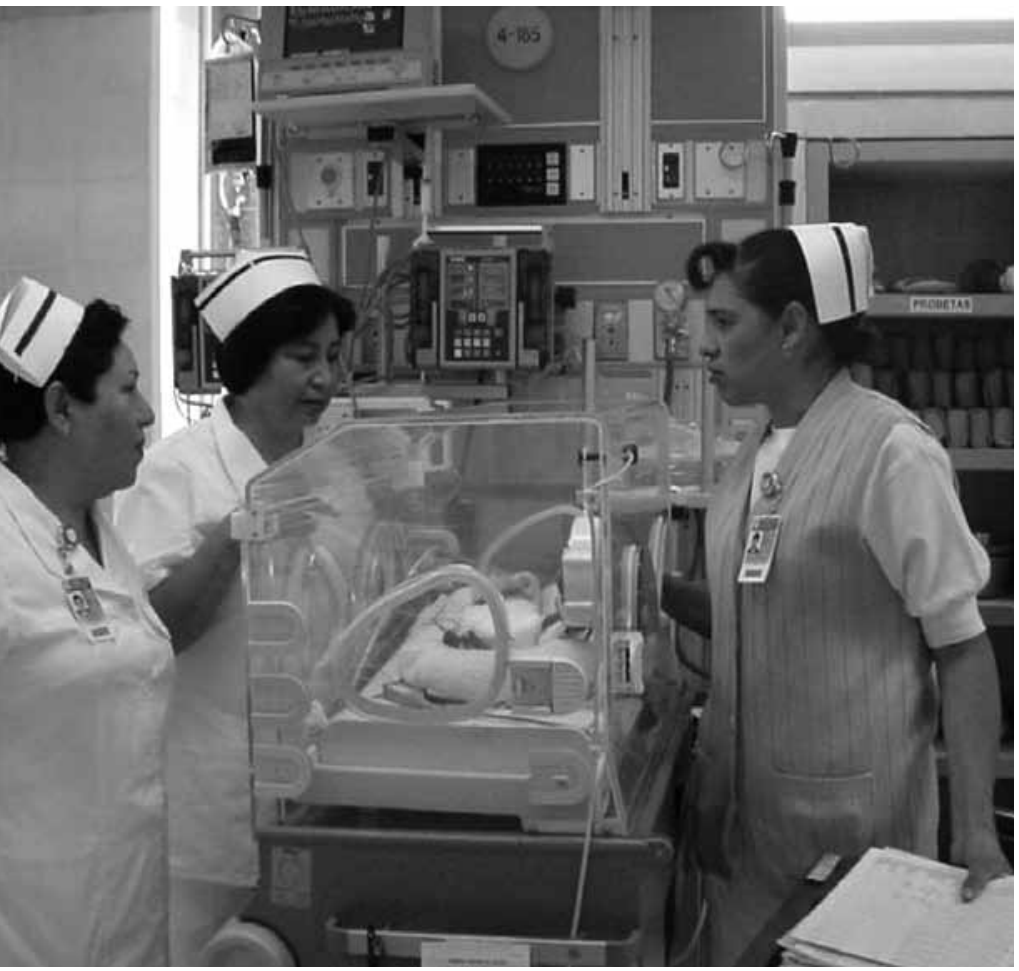

\section{REFERENCIAS BIBLIOGRÁFICAS}

1 Reynaga O. S. Educación, Trabajo, Ciencia y Tecnología: La investigación Educativa en México 1992-2002. Grupo Ideograma Editores. p.p 272

2 Navarro LM. Consideraciones teóricas para el estudio de egresados. En S. Barranco Ramson, Esquema Básico para Estudios de Egresados en Educación superior. Serie investigaciones. México: ANUIES. (1998) [En línea]. Disponible en: www.anuies.mx/anuies/libros98/lib10/0.htm. Fecha de consulta: 16 de abril, 2006.

3 Fierro, C., Fortoul, B., \& Rosas, L. (2002). Transformando la Práctica Docente: Una propuesta basada en la investigación - acción. Maestro y Enseñanza. México: Paidós.

4 Didou, A. S. (1996) Instituciones de educación superior y dinámicas laborales. Colección pedagógica universitaria. Veracruz: Universidad Veracruzana. 1996.

5 Weiss, CH. (1975)Investigación Evaluativa. Métodos para determinar la eficiencia de los programas de acción. México: Trillas.

6Fresán, M., (2000) Una propuesta para la evaluación docente en el posgrado. En: J. Loredo (coord) (2000) Evaluación de la educación docente en Educación Superior. D.F.; México: Edit. Porrúa - Universidad Anáhuac.

7 Loredo, EJ. \& Grijalva, MO. (2000). Propuesta de un instrumento de evaluación de la docencia para los estudios de posgrado. Citado por Rueda, B. \& Díaz B. Evaluación de la Docencia. Perspectivas actuales. México: Paidós Educador.

8 Morán, L. (2002). Validación de un Instrumento para Evaluación del Desempeño Docente en Estudios de Posgrado en Enfermería. Revista Desarrollo Científico de Enfermería. 10 (7)

9 Ramírez, R. y Tovar, C (2005). Egresados de las especializaciones en enfermería. Rev Colombia medica. Volumen 36 No 3 (Supl 2), 2005 (Julio-Septiembre). \{on line\} Consultado el 23-05-07en World Wide Web de: http://colombiamedica.univalle.edu.co/Nol36No3Supl1/cm36n3s1a9.htm

10 Martínez M y Salazar GB. (1999). Seguimiento de Egresados de maestría en Enfermería. Rev. Desarrollo Científico de Enfermería. Vol. 7 No. 10 Nov. - Dic.

\section{DIRECCIÓN PARA CORRESPONDENCIA}

Mtra. Martha Lilia Bernal Becerril : marthaliliabb@hotmail.com Mtra. Gandhy Ponce Gómez: gandhy_ponce@yahoo.com.mx Mtra. Laura Morán Peña: eneolm@yahoo.com.mx 